\title{
Circulating microRNAs in Cardiovascular Diseases
}

\author{
Marta Orlicka-Płocka ${ }^{*}$, Dorota Gurda ${ }^{1 *}$, Agnieszka Fedoruk-Wyszomirska', Iwona Smolarek² \\ and Eliza Wyszko ${ }^{\circledR}$
}

1Laboratory of Subcellular Structures Analyses, Department of Epigenetics, Institute of Bioorganic Chemistry Polish Academy of Sciences, Poznań, Poland; 'Department of Clinical Pharmacology, Poznan University of Medical Sciences, Poznań, Poland

\begin{abstract}
Cardiovascular Diseases (CD) are currently one of the most common causes of death. Because heart related deaths occur on such an enormous scale this phenomenon is referred to as an epidemic. Chronic and acute injury of the heart could be an effect of cardiac remodeling, which is a result of molecular, cellular and interstitial changes, influenced by hemodynamic load or neurohormonal activation (Cohn et al., 2000). These small deviations in cardiac activity and morphology may lead to an enormous negative effect. Despite a significant progress, knowledge of standard risk factors for cardiovascular diseases has become less and less effective, which is why predicting and seeking an appropriate treatment is very challenging. As a result, there is a growing interest in finding new markers of the CD. MicroRNAs (miRNAs), are short, non-coding RNAs responsible for regulation of gene expression at the post-transcriptional level. Among them that have the greatest potential are microRNA molecules that circulate in the blood plasma or serum, that are related to direct activation of signaling pathways, implicated in the aging process and thus for the development of cardiovascular disease. This paper is a summary of the current state of knowledge on miRNAs, their biogenesis and potential role as biomarkers to diagnose heart disease.
\end{abstract}

Key words: microRNA; biomarker; cardiovascular diseases; diagnostics; circulating; blood

Received: 30 May, 2016; revised: 23 June, 2016; accepted: 05 July, 2016; available on-line: 19 October, 2016

\section{INTRODUCTION}

Alterations at the molecular level in cellular function may lead to a variety of health problems, including development of a cardiovascular disease, tumorigenesis or nervous system degeneration (Bronze-da-Rocha, 2014). MicroRNAs are posttranscriptional regulators of gene expression in higher eukaryotes, playing a role in many biological processes, including cell growth, development and death (Wahid et al., 2010). According to "miRBase", it has been validated that more than 2000 mature miRNAs exist in humans (Schulte \& Zeller, 2015), with thousands of genes that may be their putative targets (Wang, 2008).

MicroRNAs exhibit tissue specificity and their misregulation, one or several of them, is associated with the development of certain diseases, such as those of the cardiovascular system. They are a key element involved in gene regulation, thus they present a potential role as biomarkers in the diagnosis of $\mathrm{CD}$, as well as a target for new therapies (Bronze-da-Rocha, 2014).
Recent reports indicate the immense potential of miRNAs circulating in blood plasma and their usefulness in the diagnosis of cardiovascular diseases. Invasive tissue biopsies could be replaced by simple and non-invasive straightforward assays applying circulating miRNAs as clinical markers, to assess a patient's health status (Gupta et al., 2010). Still, many questions remain in spite of efforts to identify more detailed mechanisms related to the tissue source and release of microRNAs into the blood.

\section{BIOGENESIS OF microRNAs}

MicroRNAs are a group of short, non-coding molecules comprised of 21-23 nucleotides (Bartel, 2004). MicroRNAs affect such processes as angiogenesis, apoptosis, and cell division and differentiation (Grenda et al., 2013). Let-7 and lin-4 were the first ever miRNAs to be identified, as they were shown to function in the normal development of a Caenorhabditis elegans (Grenda et al., 2013).

A miRNAs' sequence can be encoded within the exons, introns or untranslated regions of a gene (Isik et al., 2010). Maturation of miRNAs is a multi-step process wherein the first step is transcription. Most miRNAs arise from the primary transcripts (pri-miRNA), transcribed by RNA polymerase II or III (Wahid et al. 2010, Borchert et al., 2006). This leads to formation of the original pri-miRNA transcript (primary miRNA). Pri-miRNAs are present in the form of assembled structures, the so-called hairpins (Wang, 2008). Pri-miRNAs are processed in the nucleus by the Drosha complex (an enzyme that has an RNase III activity) and the related DGCR8 protein (Pasha; which binds to the singlestranded ends of the pri-miRNA). The cleavage product is a pre-miRNA of approximately 70 nucleotides (Okamura et al., 2009), which is transported to the cytoplasm by exportins 5 (Exp-5) interacting with the Ran GTP protein (Bartel, 2004; Min \& Chan, 2015).

An enzyme located in the cytoplasm, Dicer, that has an RNase III activity, then hydrolyzes the pre-miRNA to a double-stranded miRNA duplex approximately 21 to 23 nucleotides long. Both strands of the duplex may be functional (Okamura et al., 2009), negatively regulating expression of the target mRNA, but only the guide strand is incorporated into the RISC complex (Micro$\mathrm{RN} A$ induced silencing complex). Mature miRNA binds to a target complementary sequence in the 3'UTR region

e-mail: wyszkoe@ibch.poznan.pl

*These authors equally contributed to this work

Abbreviations: AMI, Acute Myocardial Infarction; AHF, Acute Heart Failure; CAD, Coronary Artery Disease; miRNA, microRNA; RISC, RNA Induced Silencing Complex; cTn, Cardiac Troponins 


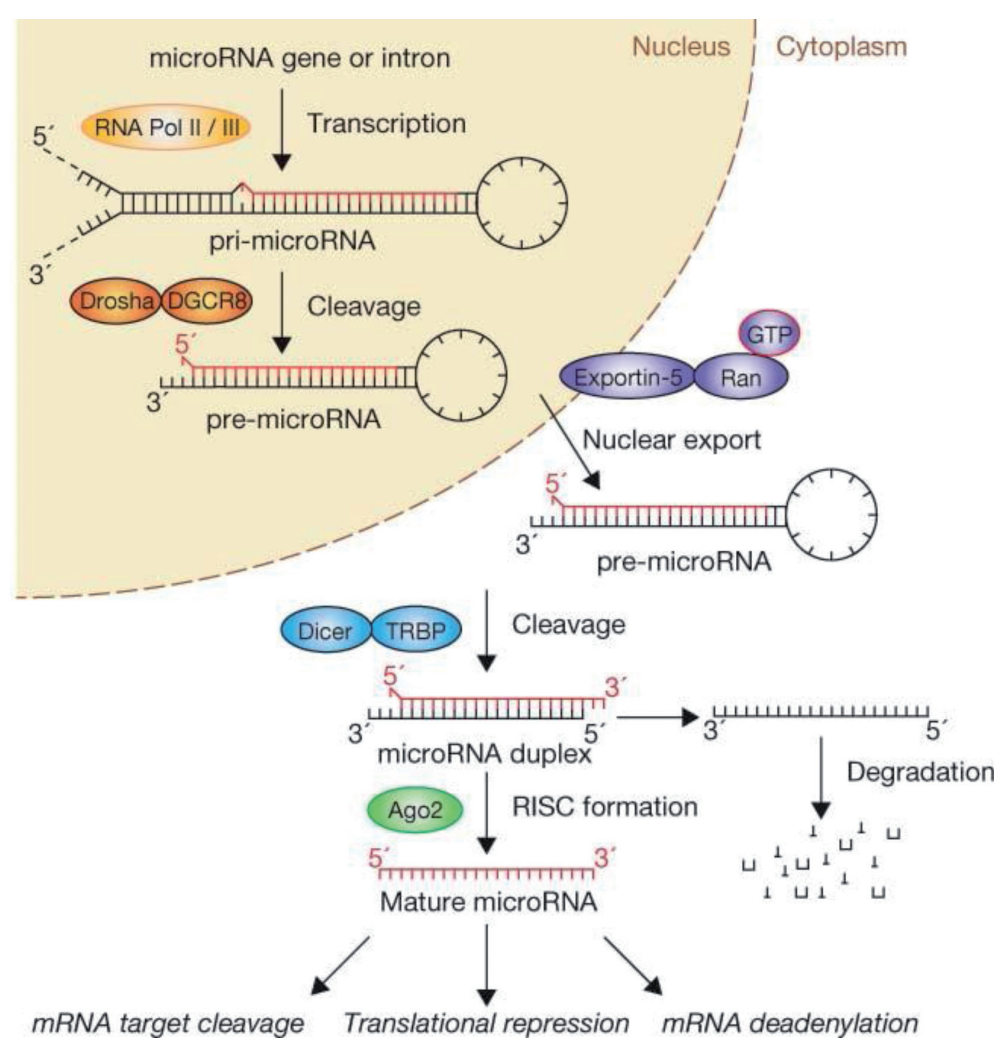

Figure 1. Schematic of microRNA biogenesis and mechanism of action. Adapted from Winter et al., 2009. .

of the target transcript (Bartel, 2004; Hrustincova et al., 2015). The discovery of miRNAs revealed their importance in many physiological processes, including cell differentiation. Gene silencing via miRNA is possible in two ways. One of them is specific mRNA degradation as a result of complete pairing of the miRNA sequence with the target mRNA's 3'UTR regions (Bartel et al., 2004). However, a second mechanism is often observed in animals, where miRNAs inhibit translation of the transcript; there, a complete pairing between target mRNA and miRNA is not essential (Bartel et al., 2004, Wahid et al., 2010). MicroRNAs' biogenesis is shown in Fig. 1.

MicroRNAs are one of the most important class of non-coding RNAs regulating gene expression in different cell types (Shiyong et al., 2010). Recently, it has been shown that a high level of miRNAs is expressed in the cells of the circulatory system: cardiomyocytes, endothelial cells and vascular smooth muscle (Urbichem et al., 2008). There have been reports on the presence of these miRNAs in the plasma and their potential as diagnostic markers of cardiovascular diseases (Laterza et al., 2009).

\section{MICRORNAS IN CARDIOVASCULAR DISEASES}

In the cardiovascular system, miRNAs play a significant role in its development and physiology. They control basic functions in all cell types of the cardiovascular system (endothelial cells, cardiac muscle, smooth muscle, inflammatory cells) and have been identified in all stages of cardiac tissue development (Romaine et al., 2015). Thus, they are crucial in the search for new solutions in diagnosis, prognosis and therapy (Condorelli et al., 2014).

After sequencing 250 small RNA libraries from different cells and tissues, a project that was com- pleted in 2007, a list of highlyexpressed and most abundant miRNAs was established. This includes miRs expressed in non-diseased cardiac tissue, among which the highest diagnostic potential have: miR-208, miR-133, miR-1, miR-16, miR-126, miR-27b and miR-143 (Landgraf et al., 2007). Some of the miRs in this group, such as miR-133 and miR-1, are related to cardiac remodeling, a process which is necessary for stress factor adaptation, but can be also a cause of myocardial infarction (Romaine et al., 2015).

Current knowledge about microRNAs emphasizes their role in both - maintenance of cardiac homeostasis and pathogenesis of cardiovascular diseases, such as Vascular diseases. They include Coronary Artery Disease (CAD) and Acute Myocardial Infarction (AMI), which are the most common causes of death in the world, and thus many studies had tried to provide insights into the significant role of miRs in their development (Pfeifer et al., 2015). In particular, miR-27b, miR-130a, miR-221 and miR-222, reg ulate angiogenesis and pathogenesis, like CAD (Schulte \& Zeller, 2015; Fichtlscherer et al., 2010). A list of the most abundant and specific miRNAs regulating the cardiac skeletal muscle metabolism has also been compiled.

So far, research has focused mainly on miR-208, miR1, miR-133, and miR-499, which are circulating in both, blood and plasma, and are directly related to the cardiac and smooth muscle cells of coronary arteries (Schulte \& Zeller, 2015). MiR-1 is considered to have pro-apoptotic properties in myocardial ischemia, while miR-133 was found to repress caspase-9 expression in the same affliction (Mitchelson \& Qin, 2015).

It was also shown that miR-208 is highly expressed in cardiomyocytes, as well as in the smooth muscle cells, and is released in AMI (Schulte \& Zeller, 2015). MicroRNA-208 (including miR-208a and miR-208b), known as myomiR, may provide a solution for a difficult detection problem, especially in the early stage of the disease (van Empel, et al., 2012). Mature sequence of miR-208 is localized in an intron of the $\alpha \mathrm{MHC}$ gene, which encodes myosin heavy chains. Proper expression pattern of the gene determines the accurate number and quality of cardiac muscle fibers (Callis et al., 2009; van Rooij et al., 2007). miR-499, which is expressed almost solely in the heart, may become another AMI biomarker,. It was shown that its level rises in the AMI patients, but is still extremely hard to detect due to its low level in the plasma samples (Adachi et al., 2010; Dimmeler et al., 2010).

Both, CAD and AMI, may constitute a cause of Heart Failure (HF), where increase of miR-23a, miR-23b, miR-24, miR-195 and miR-214 expression was observed (Pfeifer et al., 2015).

Identification of target genes and signaling pathways affecting cardiovascular system is critical for future studies, because the molecular mechanisms of cardiac fibrosis following AMI or CAD, are still poorly understood. In general, regulation of gene expression by microRNAs is based on a rule that a single miRNA can regulate ex- 
pression of several gene targets in the cells, as well as an individual gene can be regulated by many different miRNAs (Romaine et al., 2015). Different expression patterns of miRNAs may also be a result of modulation by transcription factors (Liao et al., 2015). It was discovered that miR-21 affects myocardial remodeling by regulating the ERK-MAP kinase signaling pathway of the heart fibroblasts during Heart Failure (Thum et al., 2008). The already mentioned miR-1, miR-133 and miR-208 are known regulators of genes associated with Atherosclerosis such as PIM-1 (serine/threonine- protein kinase PIM1), SP1 (transcription factor) and p21 (cyclin-dependent kinase inbibitor 1) (Romaine et al., 2015). These examples have proved that intracellular miRNAs play a key role as regulators under pathological conditions, such as cardiovascular diseases. Many studies have also demonstrated the importance of extracellular miRNAs as messengers between cells, circulating in the blood and thus comes their potential as non-invasive disease biomarkers.

\section{CIRCULATING MIRNAS AS BIOMARKERS OF CARDIOVASCULAR DISEASES}

Among heart diseases, a fast and effective diagnosis of myocardial infarction still remains the biggest challenge for doctors and researchers (Boon \& Dimmler, 2015). A sensitive, specific and noninvasive diagnostic blood test for $\mathrm{CD}$ would be very desirable, because it is difficult to obtain valid biopsies from patients.

The ideal marker of CD should be foremost characterized by high sensitivity and reproducibility, but should be also easily accessible, fast and inexpensive. Currently, the most popular biomarkers are cardiac enzymes, whose concentration increases during a heart attack. So far, the best recognized are cardiac troponins (cTn) that are present in the muscle fibers and act in regulating the striated and cardiac muscle contraction (Schulte \& Zeller, 2015). In contrast, the detection of blood circulating miRNAs appears to be more sensitive. It was shown that in the first stage of AMI (under 3 hours) the level of miR-1, miR-133 and miR-208 is raised much earlier than the c'Tn level. This observation is probably caused by faster leakage of miRNAs from the damaged cardiomiocytes than troponins. A likely reason for faster release from damaged cells is that the c'Tn are mainly bound to myofibrils, whereas miRNAs are probably bound to protein complexes in the cytosol (Wang et al., 2010; Wu et al., 1998).

MicroRNAs may be considered as putative biomarkers of the cardiovascular system because they are tissue specific, broadly accessible in the body fluids and are known to be exceedingly sensitive to disease provoked abnormalities. In 2004, the first extracellular small RNAs were discovered in the blood samples, which included let-7b, miR-21, miR15b or miR-14 (Hrustincova et al., 2015; Mitchell et al., 2008). Several years later, in 2008, miR-155, miR-210 and miR-21, were found in the plas$\mathrm{ma} / \mathrm{serum}$ of patients suffering from lymphoma (Lawrie et al., 2008). Most of the known miRNAs related to circulatory system pathologies are localized intracellularly (cardiac muscle cells, epithelial cells), whereas the highest diagnostic potential demonstrate those circulating in the blood. The level of miRNAs in the serum of healthy people is constant and easy to detect. Many studies have also attempted to characterize a panel of the circulating miRNAs in healthy individuals that might represent a baseline for comparative analyses (Hrustincova et al., 2015). This does not mean that microRNAs are released only from damaged or necrotic tissues (Fic et al., 2014).

MicroRNAs circulating in the body fluids are characterized by a higher resistance to RNases, temperature, high and low $\mathrm{pH}$, long term storage, as well as repeated freezing and thawing (Mitchell et al., 2008). Circulating microRNAs are considered to be very promising biomarkers also due to their easy detection by Real Time PCR, microarrays or more traditional methods, such as Northern blotting (Bronze-da-Rocha, 2014). However, quantitative analysis of miRNAs in bodily fluids still carries a number of disadvantages. This is primarily due to

\section{Circulating miRNA as diagnostic markers for Cardiovascular Diseases}
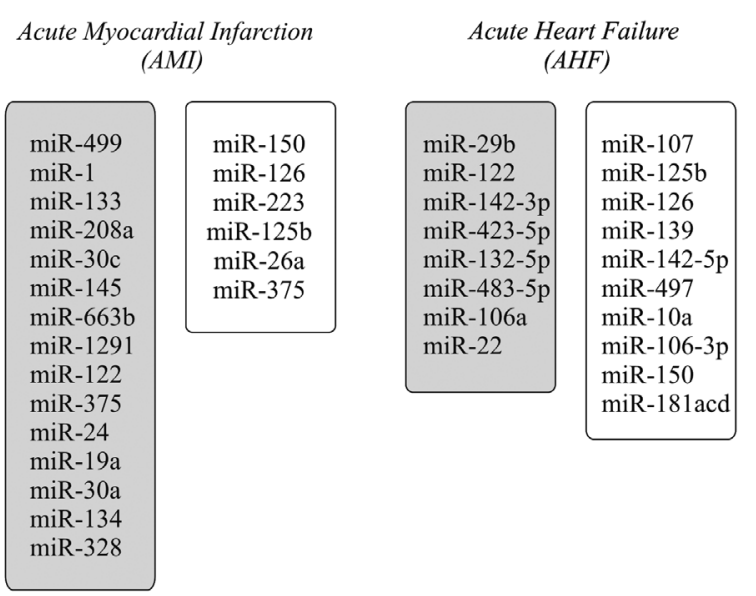

\section{Ischemic Heart Disease (IHD)}

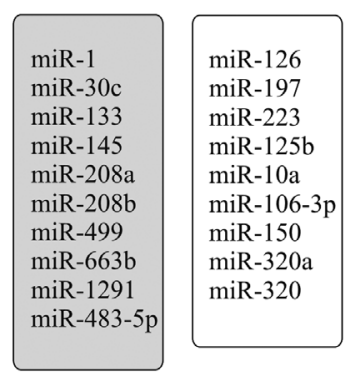

Coronary Artery Disease (CAD)

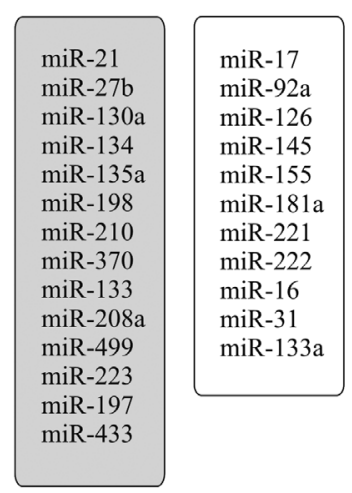

Increased expression Decreased expression 
low levels of RNA in blood or urine, which may give false positive or negative results, and requires precise standardization. Blood hemolysis is another substantial problem that may affect the accuracy of a miRNA's level of expression assessment (Hrustincova et al., 2015).

Although the precise cellular release of miRNAs into the bloodstream is not fully elucidated, several mechanisms for packaging of extracellular miRNAs for their transport were identified (Min \& Chan, 2015). MiRNAs are protected against degradation by their release in microvesicles, membrane-derived vesicle (exosomes) (Ohshima et al., 2009), apoptotic bodies (Fic et al., 2014; Min \& Chan, 2015), or associated with RNAbinding proteins (Ago2), as well as with lipoprotein complexes (HDL) (Arroyo et al., 2011; Vickers et al., 2011). Moreover, several pathways have been proposed as possible mechanisms for cellular uptake of microRNAs into the recipient tissue (Min and Chan, 2015). Blood circulating miRNAs are believed to transmit proliferation and apoptosis signals between cells (Su et al., 2015). Further studies are essential to reveal why some miRNAs are loaded into exosomes or multivesicular bodies for secretion, whereas others are kept within the cell (Creemers et al., 2012). Investigation of microRNAs in cardiovascular diseases has become more extensive, since they may be attractive diagnostic biomarkers. Figure 2, shows miRNAs circulating in the blood plasma. Their increased or decreased level, correlated with a particular disease of the circulatory system, is presented in four panels. Currently, the main target of the microRNA studies are those microRNAs whose pathological expression contributes to the development of the most common diseases, such as Acute Myocardial Infarction, Acute Heart Failure, Coronary Artery Disease and Ischemia. The precise mechanism of action for the most common circulating microRNAs are still unknown.

The usefulness of miR-1, miR-133, miR-499 and miR-208 as biomarkers for CD has been already demonstrated (D'Alessandra, et al., 2010). Differences in the expression level of individual microRNAs are often correlated with more than one particular disease, and that is why only a combination of specific microRNAs gives meaningful results in the diagnosis of Coronary Artery Disease or Acute Myocardial Infarction. The combination of their properties would probably allow to create a diagnostic test that would inform about the disease much earlier than the obvious symptoms would appear. Zampetaki et al. (2012) demonstrated that the level of miR126, together with miR-197 and miR-223, can help to specifically identify individuals with acute myocardial infarction. Other studies suggest that miR-122 along with miR-499 may constitute the basis for detection of myocardial insufficiency (Corsten et al, 2010).

The main goal for researchers is to describe the differences observed in microRNA expression profile in the blood of patients with CD and healthy people, and create diagnostic tests using a defined set of microRNAs for the diagnosis of cardiovascular diseases.

\section{CONCLUSIONS}

Finding microRNAs with the greatest diagnostic benefit should be associated with a rapid and non-invasive method of detection. Hence, interest in some miRNAs existing in the body fluids whose expression may change depending on the patient's condition is reasonable. To date, it has been shown that there is a high diagnostic potential for miR-208 and miR-499, as they appear in the blood plasma of patients with acute myocardial infarction much earlier than the cardiac troponin, the most popular biochemical AMI marker. The same microRNA molecules may be differentially expressed in the number of diseases, not only associated with the circulatory system. There are attempts to use microRNAs as therapeutic tools because of the seemingly easy way to regulate their expression. It should be taken into consideration that expression of miRNAs is not specific for a particular cell type, tissue or organ, and that caution should be taken to limit the side effects of the putative therapy. An important aim of the medical research seems to be identification and characterization of the circulating miRNAs directly related to cardiovascular diseases. MicroRNAs, through their ability to regulate gene expression, give hope for invention of new therapies (Liao et al, 2015). The relative ease of detection for microRNAs circulating in the plasma overcomes many obstacles in the diagnosis and treatment of cardiovascular diseases. In summary, future studies on microRNAs are needed to gain knowledge about their mechanism of action and effect on pathophysiological processes.

\section{Acknowledgements}

Supported by the Polish Ministry of Science and Higher Education, under the KNOW program.

\section{REFERENCES}

Adachi T, Nakanishi M, Osaka Y, Nishimura K, Hirakata G, Goto Y, Nonogi H, Iwai N (2010) Plasma MicroRNA 499 as a biomarker of acute myocardial infarction. Clin Chem 6: 1183-1185. doi: 10.1373/ clinchem.2010.144121

Arroyo J, Chevillet J, Kroh E, Ruf I, Pritchard C, Gibson D, Mitchell P, Bennett C, Pogosova-Agadjanyan E, Stirewalt D, Tait J, Tewari M (2011) Argonaute 2 complexes carry a population of circulating microRNAs independent of vesicles in human plasma. Proc Natl Acad Sci USA 108: 5003-5008

Bartel DP (2004) MicroRNAs: genomics, biogenesis, mechanism, and function. Cell 116: 281-297. doi: http://dx.doi.org/10.1016/S00928674(04)00045-5

Boon RA, Dimmeler S (2015) MicroRNAs in myocardial infarction. Nat Rev Cardiol 12: 135-142. doi:10.1038/nrcardio.2014.207

Borchert GM, Lanier W, Davidson BL (2006) RNA polymerase III transcribes human microRNAs. Nat Struct Mol Biol 13: 1097-1101. doi:10.1038/nsmb1167

Bostjancic E, Zidar N, Stajer D, Glavac D (2010) MicroRNAs miR-1, miR-133a, miR-133b and miR-208 are dysregulated in human myocardial infarction. Cardiology 115: 163-169. doi: 10.1159/000268088

Bronze-da-Rocha E (2014) MicroRNAs Espression profiles in cardiovascular diseases. Biomed Res Int 14: 1-23. http://dx.doi. org/10.1155/2014/985408

Callis TE, Pandya K, Seok HY (2009) MicroRNA-208a is a regulator of hypertrophy and conduction in mice. J Clin Invest 119: 27722786. doi: 10.1172/JCI36154

Cohn J, Ferrari R, Sharpe N (2000) Cardiac remodeling-concepts and clinical implications: a consensus paper from an international forum on cardiac remodeling. J Am Coll Cardiol 35: 569-582

Condorelli G, Latronico M, Cavaretta E (2014) microRNAs in cardiovascular diseases. J Am Coll Cardiol 63: 2177-2187. doi:10.1016/j. jacc.2014.01.050

Corsten MF, Dennert R, Jochems S, Kuznetsova T, Devaux Y,Hofstra L, Wagner DR, Staessen JA, Heymans S, Schroen B (2010) Circulating microrna-208b and microrna-499 reflect myocardial damage in Cardiovascular Disease. Circ Cardiovasc Genet 3: 499-506. doi:10.1161/CIRCGENETICS.110.957415

D'Alessandra Y, Devanna P, Limana F, Straino S, Di Carlo A, Brambilla PG, Rubino M, Carena MC, Spazzafumo L, De Simone M, Micheli B, Biglioli P, Achilli F, Martelli F, Maggiolini S, Marenzi G, Pompilio G, Capogrossi MC (2010) Circulating micrornas are new and sensitive biomarkers of myocardial infarction. Eur Heart $J$ 31: 2765-2773. doi:10.1093/eurheartj/ehq167

Fic P, Kowalczuk K, Grabarska A, Stepulak A (2014) MicroRNAs new diagnostic chances in Ischemic Heart Disease and Acute Myocardial Infarction. Postepy Hig Med Dosw 68: 410-418 (in Polish)

Fichtlscherer S, De Rosa S, Fox H, Schwietz T, Fischer A, Liebetrau C, Weber M, Hamm CW, Roxe T, Muller-Ardogan M, Bonauer A, Zeiher AM, Dimmeler S (2010) Circulating microRNAs in patients 
with coronary artery disease. Circ Res 107: 677-684. doi: 10.1161/ CIRCRESAHA.109.215566

Gan CS, Wang CW, Tan KS (2012) Circulatory microRNA-145 expression is increased in cerebral ischemia. Genet Mol Res 11: 147-152. doi: 10.4238/2012.January.27.1

Grenda A, Budzyński M, Filip AA (2013) Biogenesis of microRNAs and its meaning in etiology and process of chosen haematological disorders. Hig Med Dosw 67: 174-185 (in Polish)

Gupta KS, Bang C, Thum T (2010) Circualting MicroRNAs as biomarkers and potential paracrine mediators of Cardiovascular Disease. Circ Cardiovasc Genet 3: 484-488. doi: 10.1161/CIRCGENETICS.110.958363.

Hrustincova A, Votavova H, Dostalova Merkerova M (2015) Circulating microRNAs: methodological aspects in detection of these biomarkers. Folia Biologica 61: 203-218

Isik M, Korswagen HC, Berezikov E (2010) Expressions patterns of intronic microRNAs in Caenorhabditis elegans. Silence 1: 5 doi: 10.1186/1758-907X-1-5

Kaudewitz D, Zampetaki A, Mayr M (2015) MicroRNA biomarkers for coronary artery disease. Curr Atheroscler Rep 17: 70. doi: 10.1007/ s11883-015-0548-z

Landgraf P, Rusu M, Sheridan R (2007) A mammalian microRNA expression atlas based on small library sequencing. Cell 129: 14011414

Laterza OF, Lim L, Garrett-Engele PE (2009) Plasma micrRNAs as sensitive and specific biomarkers of tissue injury. Clin Chem 55: 1977-1983

Liao XB, de Jesus Perez VA, Król M, Yeh C, Yuan L-Q (2015) MicroRNA and cardiovascular diseases. Biomed Res Int 2015: 1-2. doi: $10.1155 / 2015 / 734380$

Meder B, Keller A, Vogel B, Haas J, Sedaghat-Hamedani F, Kayvanpour E, Just S, Borries A, Rudloff J, Leidinger P, Meese E, Katus HA, Rottbauer W (2011) Microrna signatures in total peripheral blood as novel biomarkers for acute myocardial infarction. Basic Res Cardiol 106: 13-23. doi: 10.1007/s00395-010-0123-2

Min P-K, Chan SY (2015) The biology of circulating microRNA in cardiovascular disease. Eur J Clin Invest 45: 860-874. doi:10.1111/ eci. 12475

Mitchell PS, Parkin RK, Krohn EM, Fritz BR, Wyman SK, Peterson A, Noteboom, Allen A, Urban N, Drescher CW, Martin DB, Tewari M (2008) Circulating microRNA as stable blood-based markers for cancer detection. Proc Natl Acad Sci 105: 10513-10518. doi:10.1073/ pnas.0804549105

Mitchelson KR, Qin W-Y (2015) Roles of the canonical myomiRs miR-1, miR-133 and miR-206 in cell development and disease. Worl I Biol Chem 6: 162-208

Ohshima K, Inoue K, Fujiwara A, Hatakeyama K, Kanto K, Watanabe Y, Muramatsu K, Fukuda Y, Ogura S, Yamaguchi K, Mochizuki T (2010) Let-7 microrna family is selectively secreted into the extracellular environment via exosomes in a metastatic gastric cancer cell line. PLoS One 5: 13247. http://dx.doi.org/10.1371/journal. pone.0013247

Okamura K, Liu N, Lai EC (2009) Distinct mechanisms for microRNA strand selection by Drosophila argonautes. Mol Cell 36: 431444. doi: 10.1016/j.molcel.2009.09.027

Pfeifer P, Werner N, Jansen N (2015) Role and function of MicroRNAs in extracellular vesicles in cardiovascular biology. Biomed Res Int 2015: 1-11. http://dx.doi.org/10.1155/2015/161393

Romaine S, Tomaszewski M, Condorelli G, Samani N (2015) MicroRNAs in Cardiovascular Disease: an introduction for clinicians. Heart 0: 1-8. doi:10.1136/heartjnl-2013-305402
Schulte C, Molz S, Appelbaum S, Karakas M, Ojeda F, Lau D, Hartmann T, Lackner KJ, Westermann D, Schnabel RB, Blankenberg S, Zeller T (2015) miRNA-197 and miRNA-223 predict cardiovascular death in a cohort of patients with symptomatic coronary artery disease. PLos One 10: e0145930. doi: 10.1371/journal.pone.0145930

Schulte C, Zeller T (2015) microRNA based diagnostics and therapy in Cardiovascular Disease - Summing up the facts. Cardiovasc Diagn Ther 5: 17-36. doi: 10.3978/j.issn.2223-3652.2014.12.03

Shiyong Y, Guohong L (2010) MicroRNA expression and function in cardiac ischemic injury. J Cardiovasc Transl Res 3: 241-245

Su Z, Yang Z, Xu Y, Chen Y, Yu Q (2015) MicroRNAs in apoptosis, autophagy and necroptosis. Oncotarget 6: 8474-8490.

Sun X, Zhang M, Sanagawa A, Mori C,Ito S, Iwaki S, Satoh H, Fujii S (2012) Circulating microRNA-126 in patients with coronary artery disease: correlation with LDL cholesterol. Thromb J 10: 16. doi: 10.1186/1477-9560-10-16

Thum T, Gross C, Fiedler J (2008) MicroRNA-21 contributes to myocardial disease by stimulating MAP kinase signaling in fibroblasts. Nature 456: 980-984

Urbich C, Kuehbacher A, Dimmeler S (2008) Role of microRNAs in vascular diseases, inflammation, and angiogenesis. Cardiovas Res 79: 581-588. doi: $10.1093 / \mathrm{cvr} / \mathrm{cvn} 156$

Van Empel V, De Windt L (2012) Circulating miRNAs: reflecting or affecting Cardiovascular Disease? Curr Hypertens Rep 14: 498-509

van Rooij E, Sutherland LB, Qi X, Richardson JA, Hill J, Olson EN (2007) Control of stress-dependent cardiac growth and gene expression by a microRNA. Science 316: 575-579

Vickers K, Palmisano B, Shoucri B, Shamburek R, Remaley A (2011) MicroRNAs are transported in plasma and delivered to recipient cells by high-density lipoproteins. Nat Cell Biol 13: 423-433

Wahid F, Shehzad A, Khan T, Kim Y (2010) MicroRNAs: synthesis, mechanism, function and recent clinical trials. Biochim Biophys Acta 1803: 1231-1243. doi:10.1016/j.bbamcr.2010.06.013

Wang GK, Zhu J-Q, Zhang J-T, Qing L, Li Y, He J, Qin Y-W, Jing Q (2010) Circulating microRNA: a novel potential biomarker for early diagnosis of acute myocardial infarction in humans. Eur Heart J 31: 659-666

Wang DZ (2008) An overview of microRNA. In RNA Technologies in Cardiovascular Medicine and Research. Erdmann V, Poller W, Barciszewski J eds, pp 4-12. Springer.

Winter J, Jung S, Keller S, Gregory RJ, Diederichs S (2009) Many roads to maturity: microRNA biogenesis pathways and their regulation. Nat Cell Biol 11: 228-234

Wu AH, Feng YJ (1998) Biochemical differences between cTnT and cTnI and their significance for diagnosis of acute coronary syndromes. Eur Heart J 19 (Suppl N): N25-N29

Zampetaki A, Willeit P, Tilling L, Drozdov I, Prokopi M, Renard JM, Mayr A, Weger S, Schett G, Shah A, Boulanger CM,Willeit J, Chowienczyk PJ, Kiechl S, Mayr M (2012) Prospective study on circulating micrornas and risk of myocardial infarction. $\mathrm{J} \mathrm{Am}$ Coll Cardiol 60: 290-299. doi: 10.1016/j.jacc.2012.03.056

Zeng L, Liu J, Wang Y,Wang L,Weng S, Tang Y, Zheng C, ChengQ, Chen S, Yang GY (2011) MicroRNA-210 as a novel blood biomarker in acute cerebral ischemia. Front Biosci (Elite Ed) 3: 1265-1272

Zhang C (2008) MicroRNomics: a newly emerging approach for disease biology. Physiol Genomics 33: 139-147. doi: 10.1152/physiolgenomics.00034.2008 